\title{
Effects of Ambroxol Hydrochloride on Lung Tissue Cell Apoptosis and Vascular Remodeling in Rats with Smoke Induced Chronic Obstructive Pulmonary Disease
}

\author{
JING XIE*, YEYU QIN, MIN WANG, DANNA WU, LILI ZHONG AND J. ZHANG ${ }^{1}$ \\ Department of Pharmacy, Hainan General Hospital(Hainan Affiliated Hospital of Hainan Medical University), Haikou 570311, \\ 1Department of Surgery, School Hospital, Huazhong University of Science and Technology, Wuhan 430074, China
}

Hu et al.: Effects of Ambroxol Hydrochloride

\begin{abstract}
To evaluate the effects of ambroxol hydrochloride on lung tissue cell apoptosis and vascular remodeling in rats with smoke induced chronic obstructive pulmonary disease. Sprague Dawley rats were randomly divided into normal, model, experimental and control groups $(n=20)$. The rat model of chronic obstructive pulmonary disease was established by fumigation using Yan'an Cigarettes for $64 \mathrm{~d}$. After fumigation, the experimental and control groups were subcutaneously injected with $5 \mathrm{ml}$ of Ambroxol hydrochloride (20 $\mathrm{mg} / \mathrm{kg}$ ) and $5 \mathrm{ml}$ of Bambuterol hydrochloride $(20 \mathrm{mg} / \mathrm{kg}$ ) respectively, while the normal and model groups were intraperitoneally injected with an equal dose of normal saline. Following drug intervention for 28 d, the pathological changes in lung tissues, vascular remodeling, lung tissue cell apoptosis, expressions of alpha-smooth muscle actin and vascular endothelial growth factor, as well as levels of caspase-3, B-cell lymphoma-2 and B-cell lymphoma-2 associated $X$ protein were detected by hematoxylin and eosin staining, elastic Van Gieson staining, terminal deoxynucleotidyl transferase dUTP nick end labeling staining, immunohistochemical staining and western blotting, respectively. Compared with normal group, the damage of lung tissues was obvious, the medial thickness of pulmonary arterioles significantly increased, the degree of vascular muscularization, apoptosis rate and expressions of alpha-smooth muscle actin, vascular endothelial growth factor, caspase-3 and B-cell lymphoma-2 associated $X$ protein rose and the expression of B-cell lymphoma-2 decreased in model group $(p<0.05)$. Compared with model group, the damage of lung tissues was significantly improved, the medial thickness of pulmonary arterioles decreased, the degree of vascular muscularization, apoptosis rate and expressions of alpha-smooth muscle actin, vascular endothelial growth factor, caspase-3 and B-cell lymphoma-2 associated $X$ protein reduced and the expression of B-cell lymphoma-2 rose in experimental and control groups $(p<0.05)$. Ambroxol hydrochloride can inhibit the apoptosis of lung tissue cells and improve vascular remodeling, thereby protecting the lung tissues of chronic obstructive pulmonary disease rats.
\end{abstract}

Key words: Ambroxol hydrochloride, chronic obstructive pulmonary disease, cell apoptosis, vascular remodeling

Chronic obstructive pulmonary disease (COPD) is a common chronic respiratory disease involving pulmonary vessels, pulmonary parenchyma and airways ${ }^{[1]}$. The main pathological change of frequently occurring COPD caused by smoking and air pollution is airway remodeling induced air cavity stenosis after massive inflammatory cell infiltration into peripheral and central airways and pulmonary parenchyma, so that fixed airway obstruction and pulmonary vascular wall thickening occur in patients. COPD progresses rapidly and it will easily develop into pulmonary heart disease

*Address for correspondence E-mail: 18086666325@189.cn and organ failure if not treated effectively, threatening the life ${ }^{[2]}$. At present, the nosogenesis and pathogenesis of COPD have not been fully clarified. Tsai et al. reported that pulmonary vascular remodeling induced by the apoptosis of lung tissue cells was an important

\footnotetext{
This is an open access article distributed under the terms of the Creative Commons Attribution-NonCommercial-ShareAlike 3.0 License, which allows others to remix, tweak, and build upon the work non-commercially, as long as the author is credited and the new creations are licensed under the identical terms
} 
mechanism for the occurrence and development of $\mathrm{COPD}^{[3]}$. Sun et al. showed that inhibiting the apoptosis of pulmonary capillaries can greatly inhibit the symptoms of COPD in rats ${ }^{[4]}$. Moreover, Yamada et $a l$. found that smoking led to enhanced inflammatory stress and massive apoptosis of airway epithelial cells in COPD patients ${ }^{[5]}$. Ambroxol hydrochloride is a common expectorant able to dissolve viscous sputum and lubricate the respiratory tract. Li et al. reported that ambroxol hydrochloride suppressed inflammatory stress and regulated apoptosis ${ }^{[6]}$. However, the role of ambroxol hydrochloride in vascular remodeling upon COPD has not been reported yet.

In this study, therefore, the rat model of COPD was established and the protective effect of Ambroxol hydrochloride on the lung tissues of COPD rats was explored from the perspective of apoptosis and vascular remodeling, aiming to provide valuable experimental data for the treatment of COPD.

\section{MATERIALS AND METHODS}

\section{Experimental animals, main reagents and apparatus:}

A total of 80 specific pathogen free Sprague Dawley male rats (8-10 w old, 200-220 g) were provided by Beijing Vital River Laboratory Animal Co., Ltd. (China; License No. SCXK (Beijing) 2016-0011). According to the administrative procedures for laboratory animals in our hospital, the rats were adaptively fed with standard feed and had free access to water in 4 separate cages at room temperature for $1 \mathrm{w}$. All animal experiments were approved by the Laboratory Animal Ethics Committee of our hospital (Approval No. 2019-032).

Ambroxol hydrochloride oral solution (100 ml: $0.6 \mathrm{~g}$, batch No. NMPN H20031314) was manufactured by Boehringer Ingelheim Shanghai Pharmaceuticals Co., Ltd. (China). Bambuterol hydrochloride oral solution (10 m:10 mg $\times 18$ pcs, batch No. NMPN H20051790) was manufactured by Nanjing Real Pharmaceutical Co., Ltd. (China). Yan'an Cigarettes (tar content: $10 \mathrm{mg}$; nicotine content: $1 \mathrm{mg}$ ) were produced by China Tobacco Shaanxi Industrial Co., Ltd.

Alpha-smooth muscle actin ( $\alpha$-SMA), vascular endothelial growth factor (VEGF), caspase-3, B-cell lymphoma-2 (Bcl-2), Bcl-2 associated $\mathrm{X}$ protein (Bax) and rabbit anti-glyceraldehyde-3-phosphate dehydrogenase (GAPDH) antibodies were purchased from Santa (USA). Western blotting kit was purchased from Rebstock (Germany). Immunohistochemical kit and elastic Van Gieson (EVG) staining kit were provided by Nanjing Jiancheng Bioengineering Institute (China). Hematoxylin-eosin (HE) staining kit was bought from Shanghai Beyotime Biotechnology Co., Ltd. (China). Protein concentration assay kit and diaminobenzidine (DAB) chemiluminescence kit were bought from Beijing Huaxia Yuanyang Technology Co., Ltd.(China).

LIOOS600T biological microscope was provided by Nikon (Japan). Gel imaging system was provided by Bio-Rad (USA). $-80^{\circ}$ cryogenic refrigerator was provided by WIGGENS (Germany). Leica RM2135 histotome was obtained from Leica (Germany). High speed refrigerated centrifuge was obtained from Beijing Liuyi Instrument Factory (China).

\section{Model establishment and grouping:}

After adaptive feeding for $1 \mathrm{w}$, the rats were randomly divided into normal, model, experimental and control groups $(\mathrm{n}=20)$. The rat model of COPD was established via fumigation using Yan'an Cigarettes for $64 \mathrm{~d}^{[7]}$. The normal group was fed normally at room temperature. After fumigation, the experimental and control groups were subcutaneously injected with $5 \mathrm{ml}$ of Ambroxol hydrochloride $(20 \mathrm{mg} / \mathrm{kg})$ and $5 \mathrm{ml}$ of Bambuterol hydrochloride $(20 \mathrm{mg} / \mathrm{kg})$ respectively, while the normal and model groups were intraperitoneally injected with an equal dose of normal saline. After injection, all rats were fed normally and drank water freely.

\section{Detection of respiratory function:}

Following drug intervention for $28 \mathrm{~d}$, the rats were anesthetized and fixed on an operating table in a supine position. The chest cavity was cut open using microscissors and the trachea was fully exposed, cut open in a V shape and peeled off, followed by intubation and fixation. Then HX-200 small animal breath energy exchanger and BL-420F biological functional experiment system were connected and the tidal volume, respiratory rate and minute ventilation were recorded.

\section{Observation of pathological changes of lung tissues by HE staining:}

After the respiratory function was detected, the rats were sacrificed by cardiac puncture. The chest cavity was cut open and the lung tissues were aseptically taken, $90 \%$ of which were stored in a $-80^{\circ}$ refrigerator. Subsequently, the remaining $10 \%$ of lung tissues were fixed with $10 \%$ paraformaldehyde solution for $24 \mathrm{~h}$ 
and routinely prepared into paraffin sections. Finally, the pathological changes of lung tissues were observed by HE staining under a light microscope.

\section{Observation of vascular remodeling in lung tissues by EVG staining:}

From each group, $10 \%$ of lung tissues were taken, fixed with neutral formaldehyde solution, prepared into paraffin sections, routinely deparaffinized and washed with sterile water for $10 \mathrm{~min}$, followed by EVG staining for $15 \mathrm{~min}$ and observation under an inverted fluorescence microscope. Images were analyzed using Image Pro Plus 8.0 software to evaluate the changes in the medial thickness of pulmonary arterioles and the degree of vascular muscularization ${ }^{[6]}$.

\section{Observation of lung tissue cell apoptosis by TUNEL staining:}

In each group, $10 \%$ of lung tissues were taken, routinely fixed, prepared into frozen sections, rinsed twice with xylene, rinsed with gradient alcohol for $5 \mathrm{~min}$, air dried, soaked in $3 \%$ hydrogen peroxide-methanol for $10 \mathrm{~min}$ and washed with Phosphate buffered saline (PBS) for 3 times ( $3 \mathrm{~min} /$ time). Then after pretreatment with precooled ethanol at $4^{\circ}$, the sections were treated with $0.1 \%$ TritonX-100 and $0.1 \%$ buffer for $2 \mathrm{~min}$, washed with PBS for 3 times ( $3 \mathrm{~min} /$ time), sealed with sealing film and reacted with Terminal deoxynucleotidyl transferase dUTP nick end labeling (TUNEL) mixture in a dark wet box at $37^{\circ}$ for $1 \mathrm{~h}$. Next, the sections were washed with PBS, dehydrated with gradient alcohol, transparentized with xylene and mounted with neutral rosin, followed by observation under a fluorescence microscope.

\section{Detection of $\alpha$-SMA and VEGF expressions by immunohistochemistry:}

From each group, $10 \%$ of lung tissues were taken and subjected to routine immunohistochemical staining. Brownish yellow particles under the light microscope indicated positive expression. Afterwards, the image was analyzed using Image Pro plus 8.0 software to detect the proportion of $\alpha$-SMA-and VEGF-positive cells to total cells.

\section{Detection of Caspase-3, Bax and Bcl-2 expressions by Western blotting:}

From each group, $10 \%$ of lung tissues were taken, lysed with lysis buffer and centrifuged. The supernatant was harvested, subjected to electrophoresis and incubated with the primary antibody (1:1500) and secondary antibody (1:1500), followed by color development with ELC reagent for $30 \mathrm{~min}$. After exposure, image development and fixation, the expression of target protein was analyzed using GAPDH as a reference.

\section{Statistical analysis:}

All data were statistically analyzed by Statistical package for the social sciences (SPSS) 16.0 software and figures were plotted with GraphPad 5.01 software. Intergroup comparisons were conducted by the $t$ test. $\mathrm{p}<0.05$ was considered statistically significant.

\section{RESULTS AND DISCUSSION}

The respiration curves of rats are shown in fig. 1. Compared with the normal group, the respiratory frequency was significantly increased and the ventilation and tidal volume were significantly reduced in the model group $(\mathrm{p}<0.05)$. Compared with the model group, the respiratory frequency of the experimental group and the control group decreased significantly and the ventilation and tidal volume increased significantly $(p<0.05)$. Compared with the control group, the experimental group had no significant difference, without statistical significance $(\mathrm{p}>0.05)$.

The results of HE staining are shown in fig. 2. The lung tissue of the normal group is complete, the structure of the alveolar cavity clear and the thickness of the alveolar wall uniform, without widening or exudation. No edema, inflammatory cell infiltration and exudation were found in the alveolar septum and bronchial
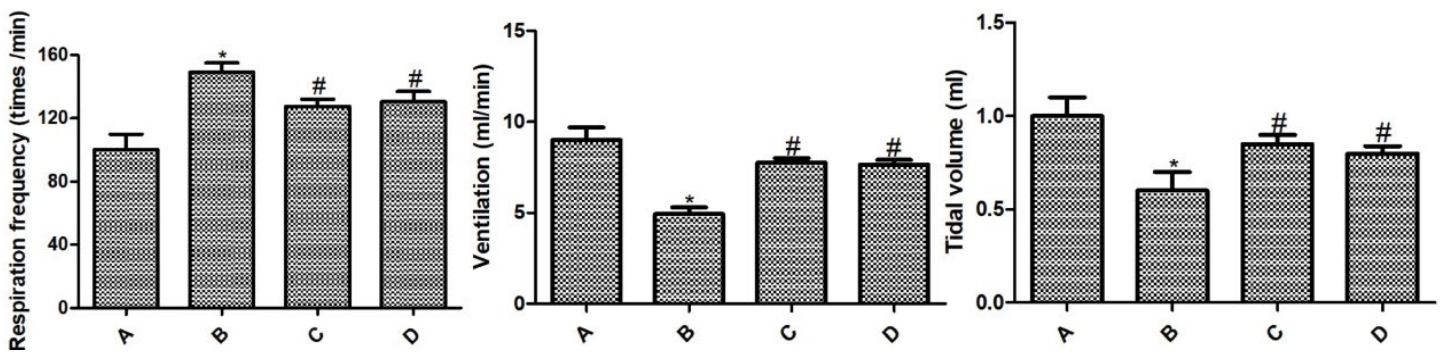

Fig. 1: Respiratory function. (A): Normal group; (B): model group; (C): experimental group; (D): control group. Compared with normal group, * $\mathbf{p}<0.05$; compared with model group, ${ }^{\#} \mathbf{p}<0.05$. 
lumen at all levels. No abnormalities were found in the structure of pulmonary artery wall. Compared with the normal group, the model group had a large amount of infiltration of inflammatory cells in the airway and around capillaries at all levels, with obvious inflammatory stress, uneven thickness of alveolar wall, narrow lumen and obvious thickening of blood vessel wall. The inflammatory stress in the lung tissues of the experimental group and the control group was significantly reduced, the pathological symptoms of the lung tissues were alleviated and the infiltration of inflammatory cells was significantly reduced.

The results of EVG staining are exhibited in fig. 3. The pulmonary arterioles in the normal group are normal in structure and the wall thickness uniform. Compared with the normal group, the media thickness of pulmonary arterioles in the model group was significantly increased and the degree of vascular muscularization was significantly increased, with statistically significant differences $(p<0.05)$. However, the media thickness of pulmonary arterioles in the experimental group and the control group was significantly reduced and the degree of vascular muscularization was significantly reduced, among which the differences were statistically significant $(p<0.05)$. Compared with the control group, the differences were not statistically significant $(\mathrm{p}>0.05)$.

The results of TUNEL staining are shown in fig. 4. Compared with the normal group, the apoptosis rate of lung tissue cells in the model group was significantly increased $(p<0.05)$. Compared with the model group, the apoptosis rate of lung tissue cells of the experimental group and the control group was significantly reduced after the drug intervention $(p<0.05)$. There was no statistically significant difference between the experimental group and the control group $(\mathrm{p}>0.05)$.

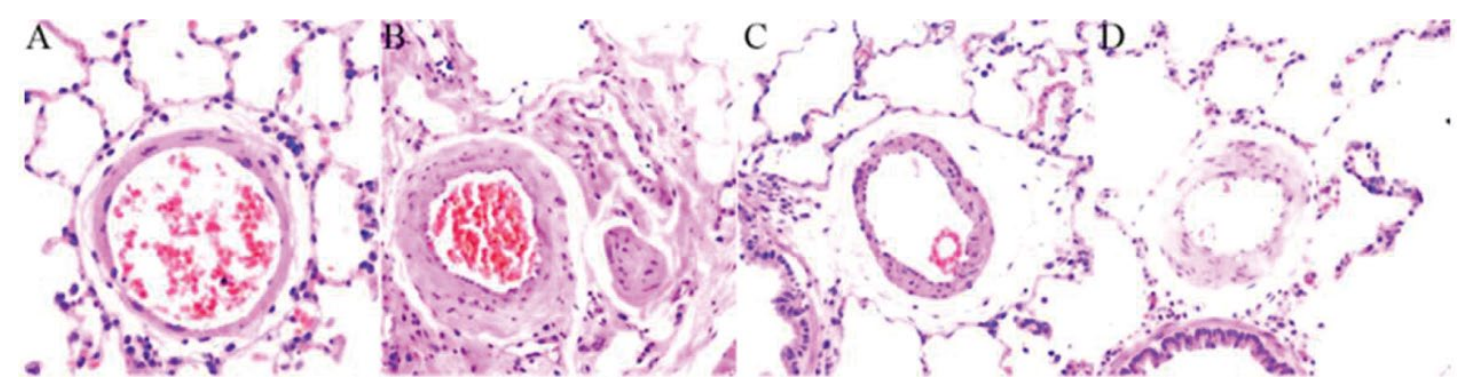

Fig. 2: HE staining results (SP×400). (A): Normal group; (B): model group; (C): experimental group; (D): control group.

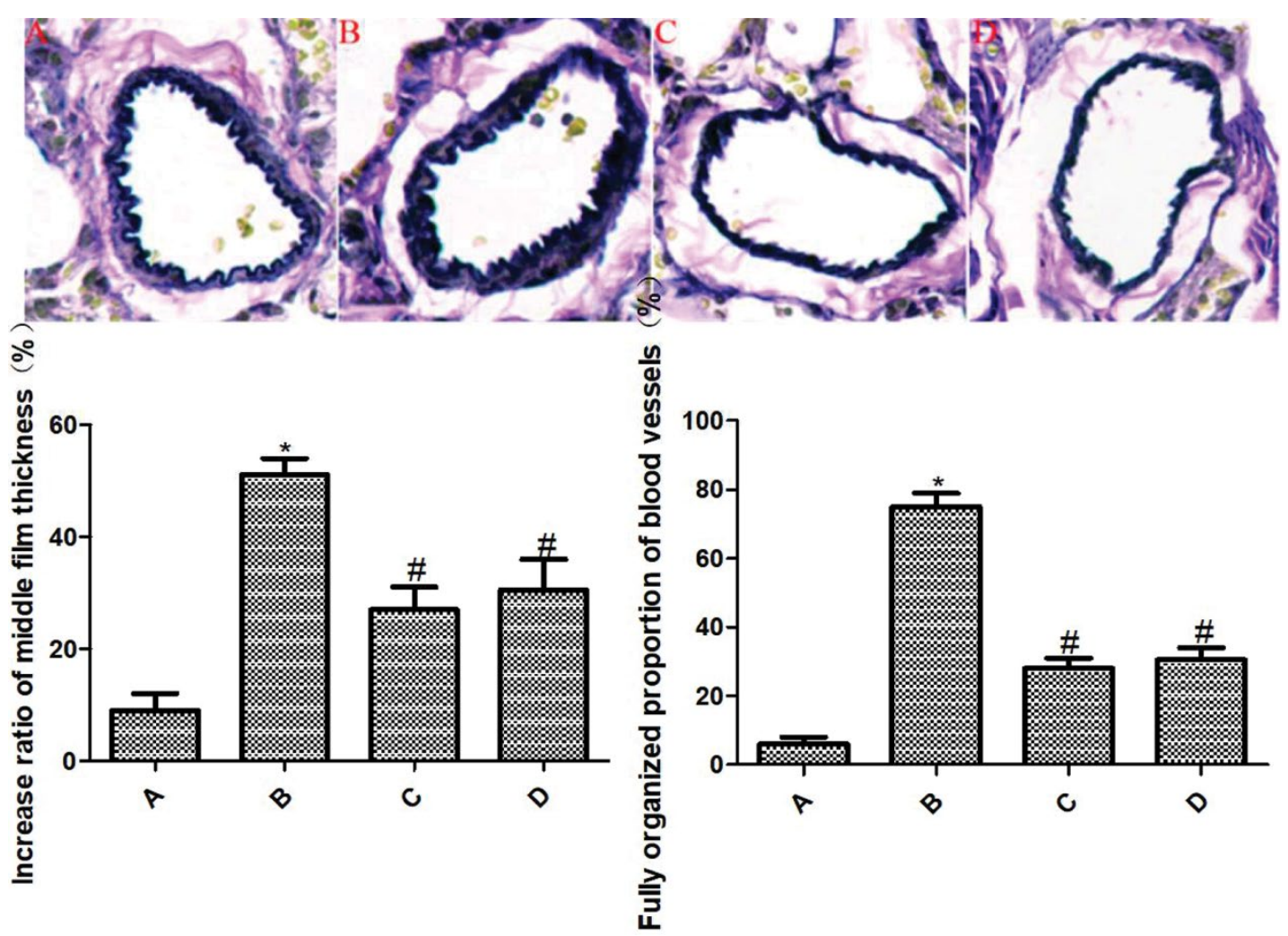

Fig. 3: EVG staining results (SP $\times 400)$. (A): Normal group; (B): model group; (C): experimental group; (D): control group. Compared with normal group, ${ }^{*} \mathbf{p}<0.05$; compared with model group, ${ }^{\#} \mathbf{p}<0.05$. 

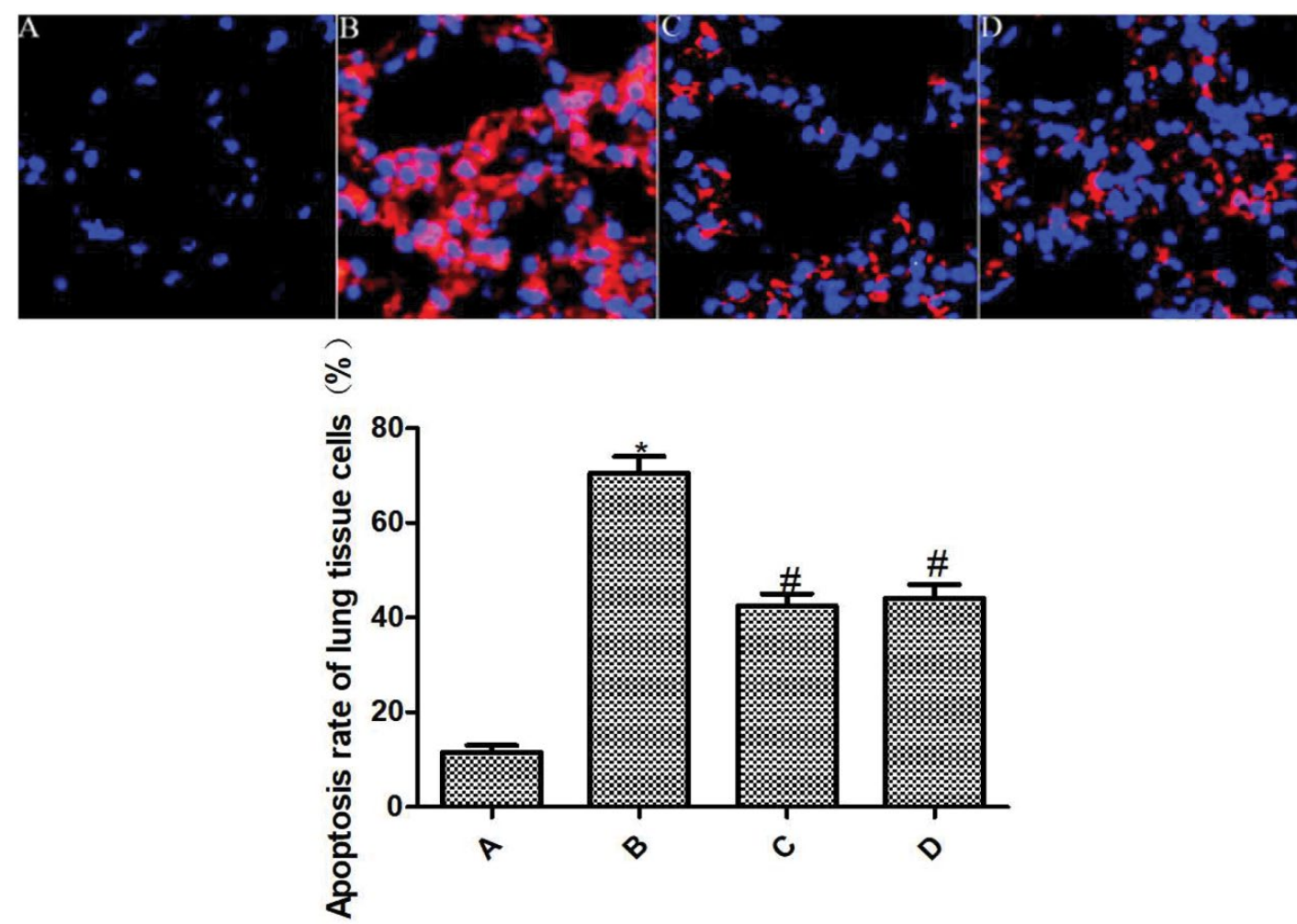

Fig. 4: Cell apoptotic rates in lung tissue detected by TUNEL assay. (A): Normal group; (B): model group; (C): experimental group; (D): control group. Compared with normal group, ${ }^{*} \mathbf{p}<0.05$; compared with model group, ${ }^{*} \mathbf{p}<0.05$.

The results of immunohistochemistry are presented in fig. 5 and fig. 6. Compared with the normal group, the expression of $\alpha$-SMA and VEGF in the lung tissue of the model group was significantly increased $(p<0.05)$. Compared with the model group, the expressions of $\alpha$-SMA and VEGF in the lung tissue of the experimental group and the control group were significantly reduced after drug intervention $(\mathrm{p}<0.05)$; the difference between the experimental group and the control group was not statistically significant $(\mathrm{p}>0.05)$.

The expressions of caspase-3, Bax and Bcl-2 in the lung tissues of rats in each group were detected by Western blot, as shown in fig. 7. Compared with the normal group, the expressions of caspase- 3 and Bax was significantly increased, and the expression of Bcl-2 was significantly reduced in the lung tissue of the model group $(p<0.05)$. Compared with the model group, the expressions of caspase- 3 and Bax were significantly reduced and the expression of Bcl-2 was significantly increased in the lung tissues of the experimental group and the control group $(p<0.05)$; the difference between the experimental group and the control group was not statistically significant $(\mathrm{p}>0.05)$

COPD is a common and frequently occurring disease. In autumn and winter in particular, COPD patients are prone to respiratory failure and even death due to acute attacks ${ }^{[8]}$. Since its pathogenesis is not yet clear, there is still a lack of rapid and effective treatment in clinical practice. Therefore, it is of great significance to clarifying the pathological mechanism of COPD and developing effective adjuvant drugs for improving the clinical treatment effect and prognosis of COPD.

Pulmonary vascular remodeling is a key event in the occurrence and development of COPD ${ }^{[9]}$. Pulmonary vascular remodeling in COPD involves the whole pulmonary vascular layer, including dysfunction of microvascular endothelial cells, phenotypic transformation of smooth muscle cells and imbalance of extracellular matrix synthesis and secretion ${ }^{[10]}$. Pulmonary vascular remodeling directly leads to pulmonary hypertension, pulmonary heart disease and right ventricular dysfunction in COPD patients, which will induce worse events if their development cannot be effectively controlled in a timely manner. Pulmonary vascular remodeling is the result of the participation of multiple factors and multiple effector cells ${ }^{[11]}$. As highly specific cytokines, $\alpha$-SMA and VEGF play an important role in the pathological process of pulmonary microvascular remodeling. $\alpha$-SMA is a key factor in the phenotypic transformation of pulmonary vascular smooth muscle cells and VEGF is an important factor that promotes the mitosis of pulmonary microvascular endothelial cells and changes vascular permeability ${ }^{[12]}$. Nielsen et al. found in clinical observations that the 


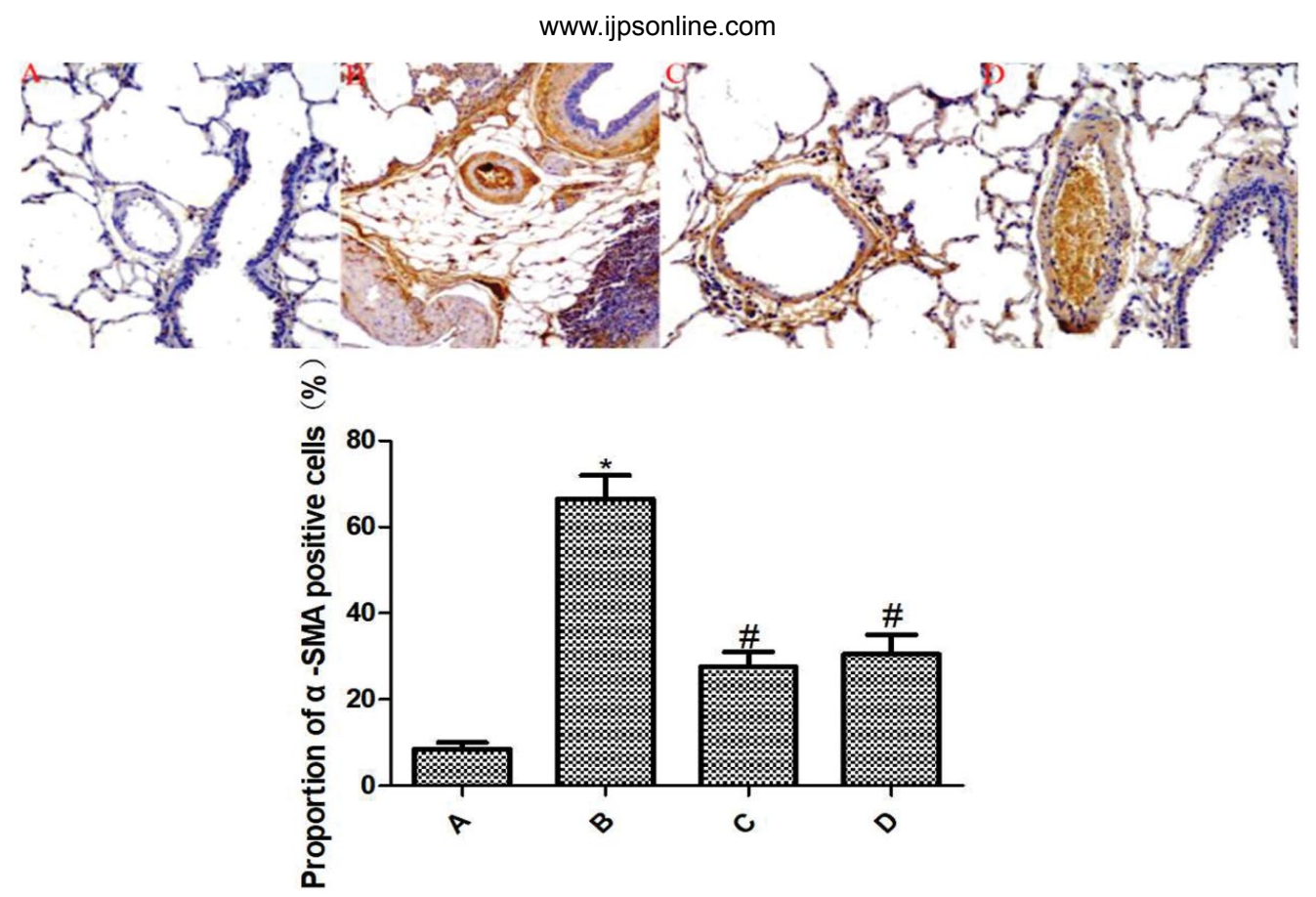

Fig. 5: $\alpha$-SMA expression in lung tissue detected by immunohistochemistry $(\mathrm{SP} \times 400)$. (A): Normal group; (B): model group; (C): experimental group; (D): control group. Compared with normal group, ${ }^{*} \mathbf{p}<0.05$; compared with model group, ${ }^{\#}<<0.05$.

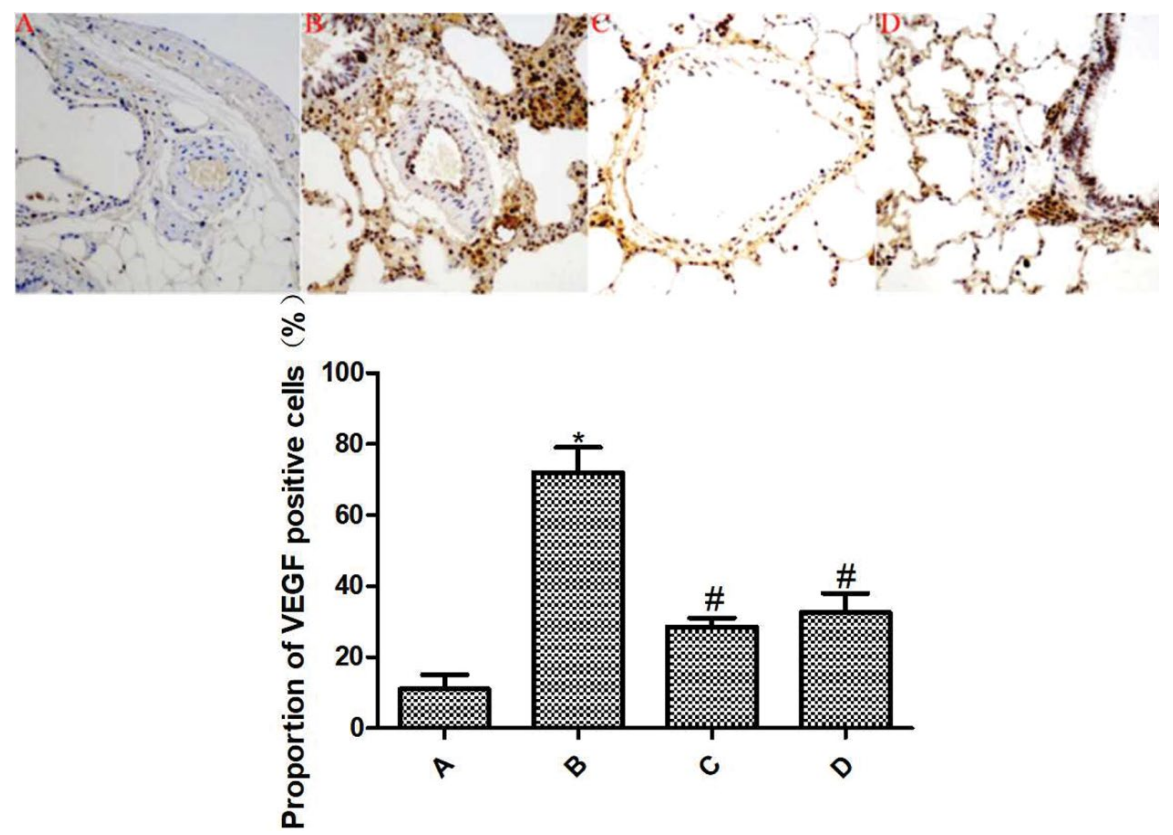

Fig. 6: VEGF expression in lung tissue detected by immunohistochemistry (SP $\times 400)$. (A): Normal group; (B): model group; (C): experimental group; (D): control group. Compared with normal group, ${ }^{*} \mathbf{p}<0.05$; compared with model group, ${ }^{\#} \mathbf{p}<0.05$.

expression level of $\alpha$-SMA was an important marker to measure the degree of pulmonary fibrosis in patients with idiopathic pulmonary fibrosis and $\mathrm{COPD}^{[13]}$. Szucs et al. reported that the increased expression of VEGF directly activated multiple signals in pulmonary smooth muscle cells and enhanced the migration and proliferation of smooth muscle cells, resulting in pulmonary artery remodeling in COPD patients ${ }^{[14]}$. Truong et al. verified that blocking the expression of VEGF can significantly improve vascular remodeling in COPD rats ${ }^{[15]}$. In this study, the media thickness of pulmonary arterioles, the degree of vascular muscularization and the expression of $\alpha$-SMA and VEGF of the model group are significantly increased, with obvious symptoms of COPD pulmonary vascular remodeling. After intervention, the three indicators of the experimental group and the control group were significantly reduced and the symptoms of COPD pulmonary vascular remodeling were significantly improved. Thus, ambroxol hydrochloride can significantly improve the pulmonary microvascular remodeling in COPD rats. 

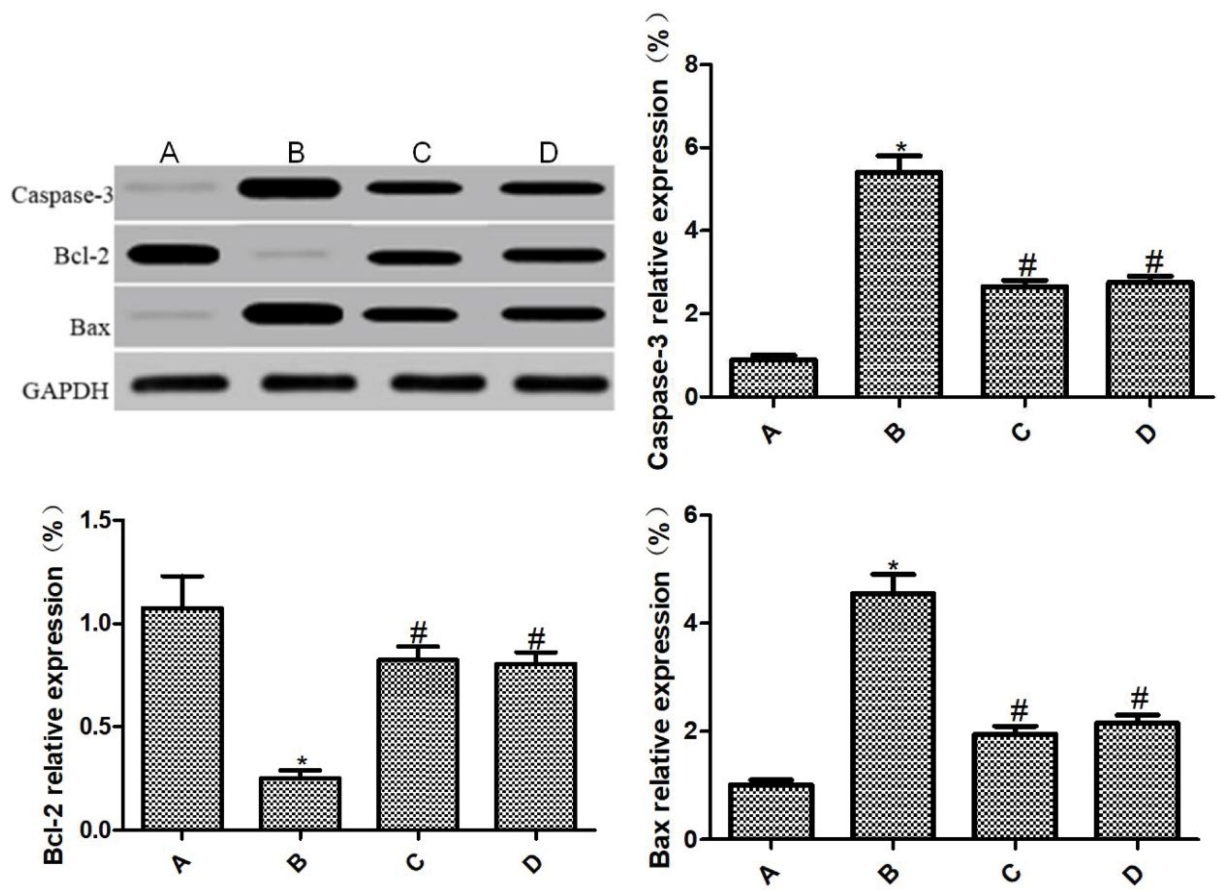

Fig. 7: Caspase-3, Bax and Bcl-2 protein expressions in lung tissue detected by Western blot. (A): Normal group; (B): model group; (C): experimental group; (D): control group. Compared with normal group, ${ }^{*} \mathbf{p}<0.05$; compared with model group, ${ }^{*} \mathbf{p}<0.05$.

The pathogenesis of COPD is very complicated. Apoptosis plays an important role in the pathogenesis of the disease $\mathrm{e}^{[5]}$. Masubuchi et al. confirmed that inhibition of apoptosis in lung tissue of COPD mice can significantly inhibit the reduction of transient potential in lung tissue and improve its symptoms ${ }^{[16]}$. Li et al. found that reducing the apoptosis rate of COPD injured mice can significantly improve the autoimmune ability of mice and shorten the recovery period ${ }^{[17]}$. Sun et al. reported that improving the apoptosis of pulmonary microvessels can significantly improve the COPD injury of patients and prevent the deterioration of lung tissue $^{[4]}$. Bcl-2 protein is the most important regulatory protein in the physiological process of apoptosis, which is mainly composed of anti-apoptotic protein $\mathrm{Bcl}-2$ and pro-apoptotic protein $\mathrm{Bax}$. Bcl-2/Bax releases signals into the mitochondria at the beginning of apoptosis, which promotes the activation of the apoptosisexecuting molecule caspase-3, leading to DNA breakage, cytoskeletal and intracellular membrane defects and even apoptosis ${ }^{[18]}$. Cho et al. pointed out that the imbalance of $\mathrm{Bcl}-2 / \mathrm{Bax}$ expression directly promoted the apoptosis of alveolar epithelial cells in COPD rats ${ }^{[19]}$. Regulating the release of $\mathrm{Bcl}-2 / \mathrm{Bax}$ signals can significantly inhibit the apoptosis of cell pairs, improve pulmonary artery pressure in COPD rats and improve the vascular remodeling of $\mathrm{COPD}^{[20]}$. Herein, in the model group, the apoptosis rate and the expressions of caspase- 3 and Bax in the lung tissue were significantly increased and the expression of Bcl-
2 was significantly reduced. After drug intervention, the apoptosis rate and the expressions of caspase- 3 and Bax in the lung tissue were obviously decreased in the experimental group and the control group and the expression of Bcl-2 was increased significantly. Hence, Ambroxol hydrochloride can inhibit the apoptosis of lung tissue in COPD rats by regulating the expression of Bcl-2/Bax.

In summary, Ambroxol hydrochloride can inhibit the apoptosis of lung tissue, improve its vascular remodeling and protect the lung tissue of COPD rats. Nevertheless, whether Ambroxol hydrochloride can achieve the same satisfactory therapeutic effect in the clinical treatment of COPD requires further research.

\section{Acknowledgement:}

This study was financially supported by Grant No. 81803018.

\section{Conflict of interests:}

The authors declared no conflicts of interest.

\section{REFERENCES}

1. Marcelino MY, Fuoco NL, Faria CA, Kozma RD, Marques LF, Ribeiro-Paes JT. Animal models in chronic obstructive pulmonary disease - an overview. Exp Lung Res 2014;40(6):259-71.

2. Valley TS, Sjoding MW, Ryan AM, Iwashyna TJ, Cooke $\mathrm{CR}$. Intensive care unit admission and survival among older patients with chronic obstructive pulmonary disease, heart failure or myocardial infarction. Ann Am Thorac Soc 
2017;14(6):943-51.

3. Tsai MJ, Chang WA, Jian SF, Chang KF, Sheu CC, Kuo PL. Possible mechanisms mediating apoptosis of bronchial epithelial cells in chronic obstructive pulmonary disease-a next-generation sequencing approach. Pathol Res Pract 2018;214(9):1489-96.

4. Sun Y, An N, Li J, Xia J, Tian Y, Zhao P, et al. miRNA-206 regulates human pulmonary microvascular endothelial cell apoptosis via targeting in chronic obstructive pulmonary disease. J Cell Biochem 2019;120(4):6223-36.

5. Yamada K, Asai K, Nagayasu F, Sato K, Ijiri N, Yoshii N, et al. Impaired nuclear factor erythroid 2-related factor 2 expression increases apoptosis of airway epithelial cells in patients with chronic obstructive pulmonary disease due to cigarette smoking. BMC pulmonary medicine 2016;16(1):1-0.

6. Li X, Wu X, Gao Y, Hao L, Sun S. Apoptosis-linked antifungal effect of ambroxol hydrochloride by cystolic calcium concentration disturbance in resistant Candida albicans. Sci China Life Sci 2019;62(12):1601-4.

7. Li MX, Chen YH, Liao CC, Lin F, Bai Y, Mi WJ, et al. Role and mechanism of hydrogen sulfide in cigarette smoke induced chronic obstructive pulmonary disease related pulmonary vascular remodeling in rats. Chin Med J 2017;97(2):137-42.

8. Caramori G, Ruggeri P, Di Stefano A, Mumby S, Girbino G, Adcock IM, et al. Autoimmunity and COPD: clinical implications. Chest 2018;153(6):1424-31.

9. Tuder RM. Pulmonary vascular remodeling in pulmonary hypertension. Cell Tissue Res 2017;367(3):643-9.

10. Lin L, Hou G, Han D, Yin Y, Kang J, Wang Q. Ursolic acid alleviates airway-vessel remodeling and muscle consumption in cigarette smoke-induced emphysema rats. BMC Pulm Med 2019;19(1):1-1.

11. Sun Z, Li F, Zhou X, Chung KF, Wang W, Wang J. Stem cell therapies for chronic obstructive pulmonary disease: current status of pre-clinical studies and clinical trials. J Thorac Dis 2018;10(2):1084-98.

12. Voelkel NF, Gomez-Arroyo J. The role of vascular endothelial growth factor in pulmonary arterial hypertension. The angiogenesis paradox. Am J Respir Cell Mol Biol 2014;51(4):474-84.

13. Nielsen SH, Willumsen N, Leeming DJ, Daniels SJ, Brix $\mathrm{S}$, Karsdal MA, et al. Serological assessment of activated fibroblasts by alpha-smooth muscle actin ( $\alpha$-SMA): a noninvasive biomarker of activated fibroblasts in lung disorders. Transl Oncol 2019;12(2):368-74.

14. Szucs B, Szucs C, Petrekanits M, Varga JT. Molecular characteristics and treatment of endothelial dysfunction in patients with COPD: a review article. Int $\mathbf{J}$ Mol Sci 2019;20(18):4329-48.

15. Truong TM, Li H, Dhapare S, Desai UR, Voelkel NF, Sakagami M. Sulfated dehydropolymer of caffeic acid: In vitro anti-lung cell death activity and in vivo intervention in emphysema induced by VEGF receptor blockade. Pulm Pharmacol Ther 2017;45:181-90.

16. Masubuchi H, Ueno M, Maeno T, Yamaguchi K, Hara K, Sunaga $\mathrm{H}$, et al. Reduced transient receptor potential vanilloid 2 expression in alveolar macrophages causes COPD in mice through impaired phagocytic activity. BMC Pulm Med 2019;19(1):1-0.

17. Li X, Yang H, Wu S, Meng Q, Sun H, Lu R, et al. Suppression of PTPN6 exacerbates aluminum oxide nanoparticle-induced COPD-like lesions in mice through activation of STAT pathway. Part Fibre Toxicol 2017;14(1):1-4.

18. Jalili C, Salahshoor MR, Moradi MT, Ahookhash M, Taghadosi M, Sohrabi M. Expression changes of apoptotic genes in tissues from mice exposed to nicotine. Asian Pac J Cancer Prev 2017;18(1):239-44.

19. Cho RJ, Kim YS, Kim JY, Oh YM. Human adipose-derived mesenchymal stem cell spheroids improve recovery in a mouse model of elastase-induced emphysema. BMB Rep 2017;50(2):79-84.

20. Giri B, Afroze SH, Choudhury S, Karanjeet R, Ghamande SA, Uddin MN. Hypoxia modulates the cellular signaling in cultured ratlung micro vascular endothelial cells. BRC 2016;2(2):230-7. 